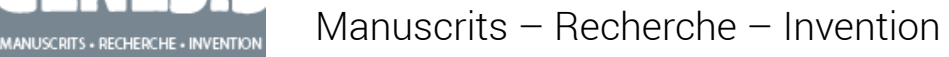

$38 \mid 2014$

Traduire

\title{
Écrire et décrire la genèse de la traduction
}

Le Désert mauve et Mercier et Camier

\section{Chiara Montini}

\section{OpenEdition}

Journals

Édition électronique

URL : http://journals.openedition.org/genesis/1290

DOI : 10.4000/genesis. 1290

ISSN : 2268-1590

Éditeur :

Presses universitaires de Paris Sorbonne (PUPS), Société internationale de génétique artistique littéraire et scientifique (SIGALES)

\section{Édition imprimée}

Date de publication : 15 avril 2014

Pagination : 85-98

ISBN : 9782840509370

ISSN : $1167-5101$

\section{Référence électronique}

Chiara Montini, «Écrire et décrire la genèse de la traduction », Genesis [En ligne], 38 | 2014, mis en ligne le 29 juin 2016, consulté le 11 juin 2020. URL : http://journals.openedition.org/genesis/1290 ; DOI : https://doi.org/10.4000/genesis. 1290 


\title{
Écrire et décrire la genèse de la traduction Le Désert mauve et Mercier et Camier
}

\author{
Chiara Montini
}

Herméneute, traducteur, théorisateur, il s'agit là de facettes d'une même activité, celle de la réception du message de l'autre1.

«

a traduction est mise en rapport, ou elle n'est rien », écrit Berman dans L'Épreuve de l'étran$g e r^{2}$. Le but de cette étude est d'interroger la genèse des traductions avec une attention toute particulière à la mise en rapport telle qu'elle s'articule entre les textes et leurs traductions. Mise en rapport qui met en jeu la relation entre le même et l'autre, entre l'écriture (comme traduction), le sujet, et bien sûr, les langues. J'aborderai la genèse de la traduction dans le sens le plus général du terme pour ensuite analyser les véritables manuscrits des traductions d'auteurs et conclure sur les brouillons des traducteurs. Qu'il s'agisse d'un auteur qui se traduit ou bien d'un traducteur « autre », la relation de la traduction au texte est toujours ambivalente et passe de l'amour fusionnel à l'isolement, de l'identification à l'éloignement, de la dépendance à l'autonomie. Mais la traduction joue aussi un rôle important sur le texte qui, grâce aux nouvelles connotations et aux nouveaux signifiants, s'enrichit et peut continuer à exister.

Aux fins de mon analyse, j'ai choisi d'étudier deux textes qui concernent à la fois à la traduction et à sa genèse : Le Désert mauve de Nicole Brossard et Mercier et/ and Camier de Samuel Beckett. Il s'agit d'un choix hasardeux, mais justifiable du fait que les deux romans proposent une fiction de l'acte du traduire (une translatio in fabula, pourrait-on dire $^{3}$ ) tout en interrogeant sa genèse. Les deux romans proposent deux approches de la traduction d'abord à travers une position théorique, idéale, qui résulte de la lecture du Désert mauve, et ensuite dans sa pratique grâce à l'étude des manuscrits de Mercier and Camier.
Dans les deux textes, genèse et traduction représentent les multiples facettes, les multiples origines de l'écriture à travers ses métamorphoses et ses possibilités. Comment le traducteur représentera-t-il l'ambiguïté du texte qui s'inscrit dans sa genèse et dans sa traduction au point de constituer un tout unique ? C'est la genèse des traductions italiennes, Il deserto malva, de Elena Basile ${ }^{4}$, et Mercier $e$ Camier de Chiara Montini, qui aidera à répondre à cette question.

\section{Écriture, genèse et traduction}

Dans Le Désert mauve aussi bien que dans Mercier et/ and Camier, que je regroupe comme s'il s'agissait d'un texte unique, le travail génétique permet d'articuler les différentes phases qui caractérisent la mise en rapport entre le texte et la traduction. Le Désert mauve rassemble le texte, la genèse en tant qu'interprétation et origine de la traduction, et la traduction véritable. Il s'agit d'une fiction, car la

1. Jean Laplanche, Entre séduction et inspiration : l'homme, Paris, Presses universitaires de France, coll. «Quadrige », 1999, p. 255.

2. Antoine Berman, L'Épreuve de l'étranger, Paris, Gallimard, 1984, p. 16. Voir aussi le commentaire de Barbara Godard à ce sujet : <www. erudit.org/revue/ttr/2001/v14/n2/000569ar.pdf>.

3. Voir, à ce sujet, Translatio in fabula, Sophie Klimis, Isabelle Ost et Stéphanie Vanasten, Bruxelles, Presses des Facultés universitaires SaintLouis, 2010.

4. Il deserto malva, traduction et préface par Elena Basile, Bari, WIP Edizioni, 2010 
traduction et sa « genèse » sont le résultat d'une réflexion de l'auteure en une seule langue. Mercier et Camier est une étape de la genèse infinie d'un roman qui semble trouver sa complétude dans le passage qui conduit à son autotraduction : Mercier and Camier.

\section{Le Désert mauve de Nicole Brossard : genèse fictive d'une traduction fictive}

Écrit en 19875 , Le Désert mauve est un roman contemporain divisé en trois parties, où Brossard imagine la genèse de la traduction d'un récit. La première partie, « Le Désert mauve », s'ouvre sur une couverture avec le titre, le nom de l'auteure fictive, Laure Angstelle, et celui de la maison d'édition censée avoir publié ce texte, les Éditions de l'Arroyo ; la deuxième, «Un livre à traduire », décrit la genèse de la traduction de ce récit, le travail mental de la lectrice et future traductrice ; la troisième, « Mauve l'horizon », qui s'ouvre sur une nouvelle couverture (avec le nom de l'auteure, suivi par le titre, le nom de la traductrice et de la maison d'édition, les Éditions de l'Angle), est la version traduite par Maude Laures, elle aussi fictive. Le livre est donc une mise en fiction de la genèse d'une traduction qui comprend aussi la traduction elle-même. Ce n'est pas un hasard si l'épigraphe cite un extrait tiré de $S i$ par une nuit d' hiver un voyageur : « Lire, c'est aller à la rencontre d'une chose qui existe mais dont personne ne sait encore ce qu'elle sera 6 . » Car, comme Calvino, qui, dans ce roman oulipien, imagine sa lectrice modèle, Brossard, elle, imagine sa traductrice modèle.

Et ce n'est pas un hasard non plus que cette auteure - qui a écrit pour la revue féministe Tessera et a été traduite, entre autres, par une de ses fondatrices, Barbara Godard - se pose la question de la traduction. Écrivaine, Canadienne, francophone, féministe engagée, Brossard a suivi toutes les traductions de ses textes vers l'anglais, ainsi que $I l$ deserto malva. Sa démarche réunit la théorie à la pratique, selon le principe de la fiction théorique du mouvement féministe canadien, par un détour fort original. Car pour elle, la traduction ne passe pas forcément par une langue « autre », mais par un sujet « autre », en l'occurrence la lectrice/traductrice qui, en dehors de la fiction, est cependant l'auteure elle-même.

La partie centrale du Désert mauve, «Un livre à traduire », n'est en réalité que la fiction de la genèse de la traduction.
Elle permet, en tant que genèse, de suivre le déroulement qui caractérise la « mise en rapport » entre « Mauve l'horizon » de Maude Laures et « Le Désert Mauve » de Laure Angstelle. En ce sens, « Un livre à traduire » représente une véritable déconstruction et reconstruction du texte qui aboutit à la traduction. «Un livre à traduire » déroule, en plusieurs étapes, les états d'une genèse : la première est centrée sur la traductrice et ses atermoiements ; la deuxième sur les « lieux et objets »; viennent ensuite les « personnages »; puis les « scènes »; les « dimensions »; et retour à la traductrice, notamment à ses manuscrits, avec notes et recommandations sur l'attitude à garder vis-à-vis du texte, de la traduction et de l'auteure. Le dernier chapitre : «Un livre à traduire/suite », décrit le manuscrit véritable :

\begin{abstract}
Il y avait maintenant plus d'un an que Maude Laures préparait son manuscrit. [...]

Maude Laures retourna à sa table de travail, prit le livre, enleva l'élastique qui retenait les pages décollées. Toutes les pages étaient annotées, ici le bleu polysémie, le vert piste sonore, le rouge à vérifier, le noir incompréhensible, le jaune familier, le rose quel genre?, le mauve quel temps? Dans les marges des attentions qui pouvaient passer pour des remarques à la mine. Parfois un dessin pour faciliter la représentation (p. 197). « au bas de la page éliminer tous les comme si possible. »
\end{abstract}

Le manuscrit traduit les élucubrations mentales de Maude Laures en couleurs signifiantes à travers lesquelles se lisent ses pensées, ses images, ses désirs, ses peurs, son imagination, ses blocages et ses doutes face au texte et à sa tâche. On voit alors se tisser le travail d'interprétation préalable et nécessaire à la traduction. Ce travail serait aussi celui d'une « traduction responsable », d'après Spivak :

There is no reason why a responsible translation should take more time in the doing. The translator's preparation might take more time, and her love for the text might be a matter of a reading skill that takes patience. But the sheer material production of the text need not be slow ${ }^{7}$.

5. Nicole Brossard, Le Désert mauve, Montréal, L'Hexagone, 1987. 6. «Leggere è andare incontro a qualcosa che sta per essere e ancora nessuno sa cosa sarà », Italo Calvino, Se una notte d' inverno un viaggiatore, Torino, Einaudi, 1979.

7. Gayatri Chakravorty Spivak, «The politics of Translations », dans Lawrence Venuti (dir.), The Translation Studies Reader, New York, Routledge, 2010, p. 369-388. 
Et ce travail, prémices nécessaires à la mise en rapport de la traduction au texte, dessine la « trajectoire » qui mène à la « substitution » de l'original :

Trajectoire, pensait Maude Laures, trajectoire. Et elle se faisait de plus en plus à l'idée de devenir une voix autre et ressemblante dans l'univers dérivé de Laure Angstelle. [...] Elle serait seule dans sa langue, alors il y aurait substitution (p. 204).

« Trajectoire » donc, et ensuite « substitution » marquent la rencontre entre la traductrice et le texte, rencontre qui passe par l'identification et ensuite par l'éloignement (nécessaire). Au travers de ces deux étapes, « une voix autre et ressemblante »s'affirme. La traduction véritable est alors «palimpseste » : « Le mauve se décomposa, fut recomposé, palimpseste dans ses yeux, un air » (p. 198).

La genèse, telle qu'elle est imaginée par Brossard, lie le texte et sa traduction, l'auteure à la traductrice, dans un rapport déséquilibré :

- Je peux vous reprocher ce qui existe dans votre livre.

- De quel droit ?

- De vous lire me donne tous les droits.

- Mais traductrice, vous n'en avez aucun. Vous avez choisi la tâche difficile de lire à rebours dans votre langue ce qui dans la mienne coule de source (p. 92).
Le dilemme entre la source et la cible, évoqué ici, souligne encore le caractère à la fois autonome et dépendant du traducteur qui voudrait avoir des droits, mais qui, d'après l'auteur, n'en a aucun. La "réalité » est dans la pratique, elle se situe quelque part entre ces deux positions. C'est dans le devenir " autre » que le texte s'éloigne et qu'il peut y avoir réécriture, palimpseste. Un nouveau texte se dessine alors inévitablement par-dessus celui qui l'a précédé et dont les traces demeurent présentes. Telle une nouvelle couche d'écriture, la traduction remplace le texte tout en lui étant redevable. De plus, elle garde un rapport tellement étroit au premier qu'il en devient la continuation, voire le complément. Ainsi la genèse met-elle en évidence la manière dont la relation se tisse - le parchemin dans sa transformation. Dans l'univers du Désert mauve, écriture et traduction convergent à travers la genèse de la traduction.

À titre d'exemple, je propose un passage crucial du roman, celui où la tragédie se dévoile, afin de mettre en parallèle «Le Désert mauve » et sa traduction « Mauve l'horizon ». Le dilemme le plus difficile à accepter pour la traductrice Maude Laures est la mort d'Angela Parkins, le véritable accident du roman :
Chaque muscle, chaque nerf, chaque cellule tient lieu de musique dans nos corps absolument. Puis le corps d'Angela Parkins remue lentement. Tout son corps est attiré vers le bas. Son corps est lourd entre mes bras. Mes bras sont lourds du corps d'Angela Parkins. Il n'y a plus de musique. La sueur d'Angela Parkins contre ma tempe. La sueur sur ma main. Angela, le silence est cru. Angela ! Un tout petit dessin sur la tempe, un tout petit trou, ocelle, Angela, nous dansons, yes ? Angela Parkins n'a plus de hanches, plus d'épaules et de nuque. Elle se dissipe. Les yeux d'Angela, vite les yeux. Il n'y a plus d'équilibre entre nous. Tout mon corps est devant le désastre. Plus un son. L'agitation tout autour comme dans un film muet. Au fond de la salle, il y a le regard impassible de l'homme long. Le désert est grand. Angela Parkins est allongée, là, exposée à tous les regards. Angela se dissipe dans le noir et le blanc de la réalité. Que s'est-il passé ? C'était pourtant un homme de génie. Of course Mélanie is night teen (Le Désert mauve, p. 74-75).
Encore un temps, une musique, nous dansons allègrement. Puis le corps d'Angela Parkins bouge si peu. Je resserre l'étreinte à la taille. Il fait chaud entre nous, sur les tempes, dans les cheveux. Angela, nous dansons ? Plus d'écho, plus de musique. Nos corps ne tiennent plus ensemble. Le silence est cru. Les yeux, vite les yeux ! La pupille grand œuvre du désir se fane. Des bruits de chaise, l'agitation, des voix qui portent. Le ravage est grand. L'hom'oblong regarde devant lui, complètement détaché de la scène. Angela Parkins est allongée sur le bois blond de la piste, le corps à tout jamais inflexible exhibé, point de mire. Mélanie, fille de la nuit, que s'est-il donc passé ? (Le Désert mauve, p. 248-249) 
Ce qui frappe immédiatement c'est que la traduction contient moins de détails que l'original et notamment moins de détails concernant la relation fusionnelle de deux corps, celui de Mélanie, la narratrice, et celui d'Angela Parkins. Cependant, tout en étant plus synthétique, la traduction s'enrichit d'une nouvelle connotation, d'un nouveau signifiant qui témoigne aussi du travail herméneutique de Maude Laures. La traductrice intervient sur le texte, en tant qu'observatrice d'abord puis participante : elle exprime son incompréhension, en même temps que son désir de comprendre en s'adressant directement à la narratrice du récit : "Mélanie, fille de la nuit, que s'est-il donc passé ? »C'est le désir de la traductrice qui intervient ici en modifiant le texte. Mais c'est aussi la « faille » du texte et donc l'échec de l'interprétation, qui permet d'y déceler plusieurs significations ou aucune. Pour Laplanche cet échec d'interprétation est aussi à l'origine du refoulement ${ }^{8}$. Car le message traduit n'est que la partie consciente du Moi. Et c'est sur cette conscience que Brossard insiste, effarée, presque, par les possibilités qu'offre la traduction de faire ressortir et de faire coexister de nouvelles subjectivités inconnues. Pour elle, la traduction est dévoilement de ce que l'auteur ne voulait pas forcément «faire passer » :

J'ai consacré toute ma journée à lire la traduction anglaise de L'Amèr dont Barbara Godard vient de m'envoyer la version finale. Travail épuisant que celui de la lecture en traduction de l'un de ses propres textes. Épuisant parce qu'aux opérations mentales que l'on exécute en rédigeant le texte, s'ajoute un processus que j'appellerais, le dévoilement. Car ce que nous choisissons de cacher dans un texte, voilà, qu'il faut maintenant le dévoiler. Là où la critique, par exemple, ne peut que présumer, rêver ou imaginer un sens à ce qu'elle lit, la traduction cherche à le certifier. C'est dans cette certification que je dois affronter ce à quoi je m'étais consciemment et scrupuleusement dérobée. Être traduite, c'est être enquêtée non pas seulement dans ce que l'on croit être mais dans sa façon même de penser dans une langue, de même que dans la façon dont nous sommes pensées par une langue. C'est avoir à s' interroger sur cette autre que je pourrais être si je pensais en anglais, en italien ou en toute autre langue. [...] La question qui se pose en traduction comme en écriture est celle $d u$ choix. Quel signifiant privilégier, élire pour animer en surface les multiples signifiés qui s'agitent invisibles et efficaces dans le volume de la conscience ${ }^{9}$ ?
L'expérience de la lecture de ses propres textes traduits par d'autres semble troubler Brossard, comme si elle n'avait plus la possibilité de se cacher ni à elle-même ni aux autres. C'est la confrontation à l'autre qui la trouble, à la façon dont l'autre reçoit le message et le traduit, en traduisant aussi ce que l'auteure n'aurait pas souhaité dévoiler. Mais c'est aussi la relation à « l'autre elle-même », celle qui aurait pu se révéler dans une autre langue. En ce sens, la traduction est aussi mise en rapport de différentes subjectivités. Le moi est « mouvant », écrit Brossard : la « genèse » de la traduction du « Désert mauve », imaginée par Brossard, est une façon d'exposer le « moi mouvant» de l'écrivaine qui se cache en même temps derrière l'auteure et la traductrice fictives. De plus, l'expérience de traduire un texte écrit par elle, dans la même langue tout en représentant la genèse de cette traduction permet de réunir les différentes options qui précèdent le choix du « signifiant » à « privilégier ». Ainsi, la genèse dévoile la véritable nature du travail d'interprétation et d'élaboration qui rend possible le passage du texte à la traduction, passage qui demande un travail préalable beaucoup plus long et émotionnellement intense que celui, plus rationnel, qui résulte dans la traduction « définitive».

En ce sens, Beckett va encore plus loin que Brossard. Car, si Brossard s'en tient à la langue française ${ }^{10}$, Beckett se confronte au changement de langue, il observe « cet autre » qui est lui-même dans une autre langue. De même que « Le Désert mauve » et « Mauve l'horizon » font partie du roman Le Désert mauve, Mercier et Camier avec Mercier and Camier entretiennent un rapport tellement étroit qu'ils pourraient constituer un seul texte. L'unité se confirme dans l'identité entre l'auteur et le traducteur, mais aussi dans le rapport que l'auteur instaure avec son texte, avec soi-même comme autre qui résulte de la genèse de l'autotraduction du roman. Mais c'est aussi ce rapport qui marque la distance, qui met en jeu la différence substantielle entre texte et traduction. Différence qui se dévoile dans les manuscrits.

8. Jean Laplanche, Entre séduction et inspiration, op. cit., p. 73.

9. Nicole Brossard, Journal intime, ou Voilà donc un manuscrit, Montréal, Les Herbes rouges, 1984, p. 22-23. Je souligne.

10. Brossard écrira plus tard La Capture du sombre, un texte, en français, où la narratrice s'imagine écrire dans une langue étrangère, Montréal, Leméac, 2007. 


\section{Genèse d'une (auto)traduction annoncée}

On n' habite pas un pays, on habite une langue ${ }^{11}$.

Écrit en français en 1947, publié en $1970^{12}$ et traduit entre 1970 et 1974, Mercier et Camier est le premier roman que Beckett rédige en français. Sans présenter Samuel Beckett et son bilinguisme ${ }^{13}$, je rappellerai simplement que c'est à partir des années quarante que l'auteur écrit tantôt en français, tantôt en anglais tout en se traduisant systématiquement dans l'autre langue. J'ai choisi d'étudier ce premier roman en français, car il fut rédigé quand le projet d'une poétique bilingue commençait à prendre forme.

Proust avait reconnu que « le devoir et la tâche d'un écrivain sont ceux d'un traducteur ${ }^{14} »$, comme si l'écriture était elle-même traduction. Beckett, qui reprend la citation dans son livre consacré à l'auteur de la Recherche ${ }^{15}$, en ajoutant : " pas un artiste, un écrivain 16 », s'en tient à ce précepte. En mettant en scène la relation du sujet au langage, à travers le voyage de Mercier et Camier, l'auteur irlandais représenterait l'écriture en tant que traduction en devenir. En effet, le voyage de Mercier et Camier, que le narrateur dit «pouvoir » raconter dans l'incipit du livre17, n'est pas un voyage d'un lieu vers un autre lieu, mais d'une langue vers une autre langue. C'est une initiation à l'écriture en langue française ${ }^{18}$. Les interrogations sur la langue, ses ambiguïtés, les incompréhensions entre les deux amis, les idiomes qui se mélangent, renforcent l'impression d'être en présence d'une langue étrangère :

1) Et le rendez-vous était à quelle heure selon toi ?

Pour le quart de neuf heures.

Je ne comprends pas.

Que ne comprends-tu pas ?

Ce que signifie le quart de neuf heures.

Cela signifie neuf heures et quart.

2) Par où on se trascine, maintenant ?

Si le premier exemple fait penser à la façon anglaise de dire l'heure (« a quarter past nine » ou «nine fifteen »), le second renvoie à l'italien «trascinarsi». L'étrangeté face à la langue représente l'étrangeté de l'auteur face à « cet autre » moi qui se dévoile dans la langue étrangère. Mais si, comme ces exemples le montrent, le texte en français contient des références plus ou moins explicites à d'autres langues, qui renverraient à un Urtext perdu et utopique, c'est grâce à la traduction en anglais que la version en français peut revendiquer sa légitimité. Car, la genèse de $\mathrm{Mer}$ cier and Camier est inscrite de façon fictive dans Mercier et Camier. Car, si Mercier et Camier contient les résumés des chapitres et des références à des passages supprimés qui font penser à un travail en cours, Mercier and Camier serait la traduction d'un texte en devenir et représenterait, en tant que tel, la continuation de ce travail, un état de la genèse.

Dans la genèse de Mercier and Camier, le mouvement de rapprochement et d'éloignement que nous avons vu en analysant le texte de Brossard a laissé des traces. En effet, le premier état des manuscrits représente la traduction littérale du texte source, il est corrigé à la main et ensuite tapé à la machine. À ce stade il existe encore une forte proximité avec le texte original qui témoigne de l'effort de « fidélité » de l'auteur à sa première version. Dans les états de genèse successifs, cependant, la traduction se détache de sa source. Des effacements de passages entiers, remplacés par des commentaires lapidaires, sont le résultat tangible du changement du rapport au texte. Les commen-

11. Émile Cioran, Aveux et anathèmes, Paris, Gallimard, coll. «Arcades », 1987, p. 21.

12. Samuel Beckett, Mercier et Camier, Paris, Les Éditions de Minuit, 1970.

13. À ce sujet je renvoie à Raymond Federman et Alan W. Friedman (dir.), Beckett translating/Translating Beckett, The Pennsylvania State University Press, 1987 ; Brian T. Fitch, Beckett and Babel. An investigation into the Status of the Bilingual Work, Toronto, University of Toronto Press, 1988 ; Pascale Sardin-Damestoy, Samuel Beckett auto-traducteur ou l'art de l' "empêchement ». Lecture bilingue et génétique des textes courts auto-traduits (1946-1980), Arras, Artois Presses Université, 2002; Chiara Montini, "La bataille du soliloque ». Genèse de la poétique bilingue de Samuel Beckett (1929-1946), New York/Amsterdam, Rodopi, 2007.

14. Marcel Proust, Le Temps retrouvé, Paris, Gallimard, coll. «Bibliothèque de la Pléiade ", vol. IV, p. 469.

15. Samuel Beckett, Proust, traduit par Édith Fournier, Paris, Les Éditions de Minuit, 1990, p. 96.

16. «The artist has acquired his text: the artisan translates it. "The duty and the task of a writer (not an artist, a writer) are those of a translator" ", Samuel Beckett, Proust, London, John Calder, 1970 (1931, Chatto and Windus Ltd.), p. 84. La citation à l'intérieur de la citation est de Proust, tandis que les mots entre parenthèses sont de Beckett.

17. Mercier et Camier, op. cit., p. 9.

18. Beckett avait déjà écrit en français auparavant. 
taires semblent proclamer l'émancipation de la traduction anglaise à l'égard du texte français, tout en affichant leur dérivation de ce dernier, auquel ils font sans cesse référence. C'est à travers des raccourcis astucieux que la traduction de Beckett s'éloigne d'abord de la version française pour instaurer avec elle un rapport dialogique et tout ironique, dans une sorte d'espace doublement ludique. Ce dialogue se présente sous différentes formes :

1) Tantôt sous la forme d'un commentaire ironique. Un long dialogue entre Mercier et Camier, par exemple, est d'abord traduit et ensuite remplacé par une phrase lapidaire qui résume la longue «altercation » entre les deux amis : «... an altercation ensued too foolish to be recorded so foolish was it » (p. 30).

2) Tantôt en tant que résumé critique où l'auteur semble ne plus comprendre ce qu'il avait voulu dire dans le texte. Dans ces cas la critique semble s'adresser aux expressions idiomatiques de la langue française, dont Beckett s'était généreusement servi dans Mercier et Camier (sans doute aussi dans une tentative de se familiariser avec cet idiome dans ce qu'il a de plus typique). Ainsi, une conversation où Camier dit à Mercier qu'il ne faut pas «brûler les étapes » ni « couper les ponts » devient : «Camier mumbled something about burnt bridges and indecent haste» (p. 41).

3) Tantôt par une ellipse : «I recapitulate, said Camier. He did, then asked his simple questions » (p. 90). «He did », ici, résume un passage d'une quinzaine de lignes traduit et ensuite effacé.

4) Tantôt par une suppression lapidaire, où l'on entend quand même l'ironie et la référence à l'autre texte. Une longue conversation entre Mercier et Camier qui discutent au sujet du parapluie, par exemple, devient dans la traduction : «Camier took the umbrella and vanished up the stairs » (p. 75). «Vanished up the stairs » serait un clin d'œil aux longues confrontations entre Mercier et Camier qui précèdent toute décision. Directe et essentielle, cette phrase s'oppose par contraste à la logorrhée de deux amis. La même démarche est adoptée pour résumer un dialogue de trois pages, d'abord traduit et ensuite à peine évoqué : «A silence ensued which Camier was the first to violate » (p. 75).

Ces exemples témoignent d'un rapport au texte qui s'est transformé au fil des quatre années nécessaires à l'établissement de la traduction ${ }^{19}$. En effet, en partant d'un souci de fidélité, l'auteur se traduisant ne peut s'empêcher d'intervenir sur son œuvre, par le biais d'une « traduction critique ». Car non seulement Mercier et Camier ne répond plus à sa nouvelle poétique où l'économie de la langue devient fondamentale, mais ce vieux texte fait aussi affleurer quelque chose que l'auteur n'aurait pas voulu montrer. C'est ce que Brossard appelle le dévoilement. Mercier et Camier reste un référent avec lequel l'auteur-traducteur dialogue, tout en s'interrogeant. En même temps la traduction « cache » une partie du texte écrit auparavant, mais en garde aussi une trace. La genèse de Mercier and Camier « raconte » comment la traduction donne légitimité à Mercier et Camier tout en l'enrichissant, graduellement, jusqu'à constituer un nouveau texte qui contient le commentaire et la glose. On pourrait supposer que c'est la raison pour laquelle Mercier et Camier n'a été publié qu'en 1970, vingt-trois ans après sa rédaction et au moment où l'auteur entreprend de le traduire.

Pour l'auteur irlandais, dont l'écriture semble ne pas dépasser le stade de la genèse et donc ne pas arriver à ce texte définitif, à l'original qui ferait autorité20, la présence d'un second rend légitime le premier ${ }^{21}$. L'auteur et l'auteur autotraducteur instaurent ainsi un rapport qui prévoit un échange, et les manuscrits de la traduction dé-

19. Je n'ai pas pu donner la traduction complète présente dans les premiers états des manuscrits pour des questions de place.

20. Non fini par épuisement de l'auteur porte-parole (Watt) ou parce que le roman sera suivi par un autre (Molloy) ou encore par la nécessité de continuer (L'Innommable) ou par l'impossibilité d'en finir (Pour en finir encore).

21. Jacques Derrida, «La scène de l'écriture », dans L'Écriture et la différence, Paris, Éditions du Seuil, coll. «Points Essais », 1967, p. 293-339. Vincent Descombes explique de façon remarquable ce processus : «Il faut dire que le premier n'est pas le premier s'il n'y a pas, après lui, un second. Par conséquent, le second n'est pas ce qui vient seulement, tel un retardataire, après le premier, mais il est ce qui permet au premier d'être le premier. Ainsi le premier ne parvient donc pas à être le premier par ses seules forces, par ses propres moyens : il faut que le second l'aide de toute la puissance de son retard. La « seconde fois » a donc une sorte de priorité sur la «première fois » : elle est présente, dès la première fois, comme la condition préalable de la priorité de la première fois (sans être elle-même, bien sûr, une «première fois » plus primitive : il s'ensuit que la «première fois » est en réalité la «troisième fois »), dans Le Même et l'autre. Quarante-cinq ans de philosophie française (19331978), Paris, Les Éditions de Minuit, 1979, p. 170. 
voilent la différence entre celui qui écrit en français dans les années quarante et celui qui se traduit dans sa langue maternelle presque trente années après. Différence qui est aussi identité, conflit, compromis, accréditant ainsi une fois de plus la traduction en tant que «mise en rapport».

\section{La genèse des traductions}

La superposition de trois différentes manifestations de l'écriture : écriture, genèse et traduction, mises en scène dans Le Désert Mauve et Mercier etland Camier rendent la tâche du traducteur quelque peu ardue. Que le texte contienne sa traduction et sa genèse ne peut être ignoré par le traducteur. Mais comment reproduire cet effet de mise en abîme ? Comment représenter la multiplicité qui résulte de ces deux textes dans une traduction? La traduction dans une autre langue peut avoir, elle aussi, un rapport de complémentarité avec le texte « original »? La traduction ne serait-elle pas une continuation, voire une nouvelle couche, de ce processus sans fin si caractéristique des textes de Brossard et de Beckett, pour ne pas dire de l'écriture ellemême?

\section{Il deserto malva, traduction en italien de Elena Basile}

«Traduire un roman qui pose comme thème structural de son échafaudage narratif la traduction elle-même n'est pas une tâche indifférente. J'ai traduit au carré 22 », c'est ainsi qu'Elena Basile commente sa tâche dans ses brouillons. En effet, les fichiers de travail de Basile révèlent une traduction expérimentale, qui naît en tant que projet de thèse, dirigée par Barbara Godard, l'une des traductrices de Brossard et cofondatrice de la revue Tessera. Ces fichiers, dont les premiers remontent à 1998, témoignent du travail minutieux d'interprétation et de dissection du texte qui précède la traduction publiée en 2011. De plus, les livres et les annotations confirment que Basile lit attentivement la traduction anglaise, se familiarise avec l'œuvre de Brossard et avec son « horizon culturel23 », pour enfin procéder à la traduction.

Le Désert mauve est souligné, marqué, annoté, parfois avec des couleurs, comme les manuscrits de Maude Laures. Basile signale ses doutes linguistiques et traductifs, transcrivant en marge de Brossard la traduction anglaise de Susanne de Lotbinière-Harwood, Mauve Desert ${ }^{24}$; ensuite elle souligne les points signifiants tout en ajoutant ses commentaires. Parfois la traduction de Maude Laures est retranscrite en marge du texte de Laure Angstelle, pour créer un parallèle nécessaire à la traduction, pour éviter les répétitions là où il n'y en a pas, et pour insister sur la différence entre les deux versions.

Les brouillons de la traduction en italien (sous forme de fichiers), en revanche, sont accompagnés de longs commentaires en anglais où Basile justifie ses choix en les expliquant à sa directrice de thèse non italophone (voir exemple ci-dessous en annexe). En ce sens, cette traduction, qui contient plusieurs états de travail et se constitue en présence d'au moins trois langues, ajoute une couche supplémentaire à celles déjà présentes dans l'œuvre de Brossard.

Dans ses brouillons, et notamment dans un fichier intitulé « Notes on the translation», Basile explicite ses intentions : elle a voulu faire comme Maude Laures, elle a écouté ses désirs. Car elle considère que pour Brossard la traduction est « un mode d'inscription du désir de la traductrice 25 ». Son désir est donc légitimé par l'image de la traductrice "modèle » créée par l'auteure elle-même. Cependant, de même que Laure Angstelle dans la conversation imaginée par Maude Laures citée plus haut, Brossard exerce une certaine autorité sur Basile. En effet, la traductrice adresse une trentaine de questions à l'auteure tout en expliquant ses choix, et c'est Brossard qui décide ce qui est « acceptable » et ce qui ne l'est pas. Par exemple, Basile réfléchit à la traduction du mot « l'hom'oblong » (traduction déformée et déformante de «l'homme long » proposé par Maude Laures) :

Pour la traduction du nom de L'homme o'blong, ma solution a été la suivante : «l'omo'blungo ». Je ne peux pas reproduire le jeu sur «l'homme au blanc », mais l'omo'blungo

22. « Tradurre un romanzo che mette a tema strutturale della sua impalcatura narrativa la traduzione stessa è compito di non poco conto. Ho tradotto al quadrato » [ma traduction].

23. Antoine Berman, Pour une critique des traductions : John Donne, Paris, Gallimard, 1995.

24. Nicole Brossard, Mauve desert, trad. Susanne de Lotbinière-Harwood, Toronto, Coach House Books, 2006 (1990).

25. «A mode of inscription of the translator's desire » [ma traduction]. 
contient une petite allusion à l'homosexualité-homosocialité de l'homme long par l'utilisation de « omo » au lieu de « uomo».

Et voici la réponse de Brossard :

L'omo'blungo me semble intéressant mais vraiment à la condition que l'allusion soit très subtile.

Basile garde sa traduction. Mais il arrive aussi que la réponse de l'auteure soit plutôt négative, comme dans le cas suivant :

J'ai traduit « fils de chiennes » (p. 17, 23) avec « figli di puttana » (fils de putain). « Figli di cagna » n'est pas une option en italien.

\section{Et Brossard de rétorquer :}

J'aurais préféré insulter les chiennes plutôt que les putains. Mais si on ne peut pas, on ne peut pas.

Basile modifie sa traduction en fonction des commentaires de l'auteure, traduction qui devient «Figli di cane».

Ces quelques exemples montrent comment le plurilinguisme latent du texte et sa multiplicité se résolvent dans une traduction multilingue et subjective qui se constitue lentement, grâce à un travail préalable d'interprétation, de construction et déconstruction, de comparaison et de confrontation. L'échange avec l'auteure souligne que, tout en laissant le choix à la traductrice, Brossard a une idée précise de ce qu'elle souhaite que Le Désert mauve devienne en traduction : la continuation et le complément de son texte tout en étant une nouvelle création.

\section{Mercier e Camier, traduction en italien de Chiara Montini}

Malgré l'apparente improvisation qui caractérise $\mathrm{Mer}$ cier et Camier, la genèse de Mercier and Camier confirme que Beckett avait, lui aussi, une idée bien précise de ce qu'il attendait d'une traduction. C'est à la sonorité et au rythme qu'il tenait tout particulièrement et, comme son traducteur allemand Elmar Tophoven le dit dans un entretien à ce sujet 26 , il pouvait modifier un mot et son sens pour respecter la musique du texte.
Traduire Mercier et Camier en italien a été pour moi le couronnement de plusieurs années de recherche sur Beckett et son bilinguisme. Les documents de genèse de ma traduction montrent que les textes en français et en anglais sont soulignés, commentés, mis en rapport et confrontés sans cesse. Ce n'est donc qu'après un véritable travail d'interprétation, de comparaison et ensuite d'appropriation que j'ai commencé à traduire. J'ai alors essayé de traduire le bilinguisme tout en regroupant plusieurs options traductives dans mes brouillons. Mes premières tentatives abondent en explications et notes et réunissent ces différentes options tout en faisant coexister les versions anglaise et française et parfois même la traduction en langue italienne qui a précédé la mienne.

Mon intention initiale, pour traduire le bilinguisme, consistait à mettre en parallèle les deux traductions - en traduisant d'abord la version française, puis l'anglaise. Cette option ne va pas sans rappeler le roman de Brossard, car ici le texte et sa traduction auraient été en italien. Mais l'éditeur refusa de s'engager dans une pareille publication. Je proposai donc une édition critique où j'aurais traduit à partir du texte en français tout en explicitant les passages où l'autotraduction anglaise s'éloigne sensiblement du premier texte. Nouveau refus de l'éditeur. Pour des raisons commerciales, ces deux solutions, trop élaborées et « encombrantes », ne faisaient pas l'affaire. Finalement, j'ai traduit à partir de deux textes, tout en privilégiant le premier, celui en français, du fait que Beckett avait accepté cette convention. Cependant, le choix n'a pas été évident et, tout au long de mon travail, la version anglaise réapparaît en bas de page dans les cas où elle pourrait donner lieu à une traduction différente ou encore dans les cas où la traduction témoigne d'une tentative de faire se rencontrer les deux langues.

Traduire Beckett c'est faire l'expérience de la multiplicité de solutions traductives, mais aussi celle de l'intraduisible. Je n'ai pu restituer le bilinguisme, en définitive, car chez Beckett le texte bilingue devient un tout unique tout en étant constitué de deux «versions ». J'ai dû choisir un texte, tout en écoutant l'autre langue, l'autre version qui

26. Elmar Tophoven, « Traducendo Beckett », traduit de l'anglais par Chiara Montini, Testo a Fronte, $\mathrm{n}^{\circ} 35$, « Samuel Beckett », dir. Andrea Inglese et Chiara Montini, Milano, Marcos y Marcos, 2006, p. 302 sq. 
agissait comme un écho sur ma traduction. Même si elle ne regroupe pas les deux textes, comme je l'aurais souhaité, car c'est le choix vers lequel mon interprétation de Mercier et/and Camier m'aurait naturellement conduite, ma traduction vise à reproduire la duplicité de l'écriture beckettienne, l'ambiguïté du sujet de cette écriture, en y ajoutant d'autres couches linguistiques et un nouveau sujet : moi en tant que traductrice. C'est dans la genèse qu'on peut voir le « devenir traduction » à travers de nombreux atermoiements, tentatives et compromis qui montrent encore une fois que la traduction est mise en rapport et que cette mise en rapport est synonyme d' « ouverture, dialogue, métissage, décentrement $\gg 27$.

\section{Conclusion : entre écriture, genèse et traduction}

Fort! Da! C'est bien déjà dans sa solitude que le désir du petit d' homme est devenu le désir d'un autre, d'un alter ego qui le domine et dont l'objet de désir est désormais sa propre peine 28 .

Laplanche voit chez l'enfant la première tentative de traduire un message énigmatique. En ce sens : « La traduction est "mise en ordre", "mise en roman", "mise en temps" et, finalement, "mise en Moi"29. » La traduction, poursuit Laplanche, demande l'intervention d'un autre sujet qui « s'efforce de faire passer (c'est le sens de traduire : transducere) un message cohérent en quelque chose qui ne soit pas moins cohérent ${ }^{30} »$. C'est ce passage, qui présuppose une « mise en », qui est illustré par la genèse des traductions. Car la genèse montre, étape après étape, strate après strate, comment la traduction enrichit le texte de nouvelles interprétations, d'éléments inédits, tout en témoignant des mécanismes sous-jacents à la «mise en ordre », à la « mise en roman », à la « mise en temps », et à la « mise en Moi ».

Non seulement alors la traduction assure la continuation du processus d'interprétation - et donc s'inscrit dans ce qui est l'évolution naturelle du texte telle qu'elle est représentée dans les travaux de Brossard et de Beckett -, mais elle se distingue en tant que nouveau texte. À ce propos, Berman se demande : «Une traduction ne vise-telle pas, non seulement à "rendre" l'original, à en être le "double" (confirmant ainsi sa secondarité), mais à devenir, à être aussi une œuvre ? » et il ajoute : « Lorsqu'elle atteint cette double visée, une traduction devient un "nouvel original 31 ". Si la traduction est palimpseste, car elle met en marche un processus de « substitution », la genèse donne visibilité à toutes les couches du parchemin, l'une après l'autre, illustrant ainsi non seulement le fonctionnement et la nécessité de la traduction comme « mise en rapport », mais en représentant aussi les mouvements de rapprochement (le « double ») et d'éloignement (le « nouvel original »), le «Fort! Da! », qui la distinguent.

Le Désert mauve et Mercier etland Camier, qui contiennent la genèse même de leur traduction dans une unité caractérisée par ses multiples « couches », filent la métaphore du moi en tant qu'oignon de Lacan : « Le moi, c'est un objet fait comme un oignon, on pourrait le peler, et on trouverait les identifications successives qui l'ont constitué32. » Or, dans l'oignon il n'y a pas de centre, mais une stratification de couches successives. La genèse de la traduction, telle qu'elle est évoquée dans les deux textes, n'a pas de centre et se constitue de plusieurs stratifications qui renvoient à l' « objet » moi-oignon. Chez Brossard, le procédé est plus explicite que chez Beckett, car les différents points de vue de l'auteure, de la traductrice et de la narratrice témoignent des différentes stratifications du moi qui ont lieu au fur et à mesure que la genèse de la traduction avance. Chez Brossard, texte et subjectivités semblent partager un même destin. Chez Beckett, l'écriture est déjà stratification, chaque phrase s'enrichit d'une nouvelle connotation tout en reprenant le discours qui la précédait, et genèse et traduction font partie de ce processus complexe constitué de plusieurs stratifications où l'objet (le récit) et le sujet (écrivant) de la narration semblent se confondre jusqu'à constituer un tout unique.

C'est d'abord la genèse, dont la traduction serait l'aboutissement, qui montre les différentes couches du texte et du moi. Dans cette optique, l' « original » et le «créateur»

27. Antoine Berman, Pour une critique des traductions, op. cit., p. 16.

28. Jacques Lacan, «Fonction et champ de la parole et du langage », Écrits I, Paris, Éditions du Seuil, 1999 (1966), p. 317.

29. Jean Laplanche, op. cit., p. 206.

30. Ibid., p. 207.

31. Antoine Berman, Pour une critique des traductions, op. cit., p. 42.

32. Jacques Lacan, Le Séminaire, livre I. Les écrits techniques de Freud (1953-1954), Paris, Éditions du Seuil, p. 194. 
si chers aux romantiques deviennent des concepts fuyants, mouvants et sujets aux aléas du temps, de la langue et du lieu. Dans le début de L'Innommable - «Où maintenant ? Quand maintenant ? Qui maintenant ?»- les repères ont été perdus, ce qui reste est le texte en mouvement qui ne s'arrête pas tant qu'il continue à se faire. Il est clair alors que la traduction dans une autre langue est aussi une stratification qui s'ajoute au texte en devenir. La génétique de la traduction monte et démonte les mécanismes à la base de ce processus tout en enrichissant la traduction de nouvelles possibilités.

\section{Annexes - Quelques extraits des genèses}

Avant que le message à transmettre par le traducteur « autre » devienne cohérent, on l'a dit, de longues démarches de réflexions et recherche ont précédé la véritable traduction. Je montrerai ici respectivement deux exemples de la genèse de Il deserto malva et de Mercier e Camier.

\section{Il deserto malva, Elena Basile}

\section{1) Notes On The Translation}

If we understand translation - as Brossard does in her novel - as a mode of inscription of the translator's desire, this particular translation indeed bears witness to my own. To translate a text that has translation as its central theme and operating mechanism is a vertiginous task. As an interlingual translator I encounter in the text a "mise-en-abîme" of my own function, in that my relation to Nicole Brossard is mirrored in her book through the relation between Laure Angstelle (author) and Maude Laures (translator). The text mediates desire. Its story stages the enigma of a woman's death, and records the cognitive and affective struggle that another woman (the reader/translator) goes through in order to account for this death in her own words, in her own language.

> If Maude Laures struggled with the difficulty of accepting the textual reality of Angela Parkins' death - and her translation testifies to such a struggle - my translation struggles with the unexpected possibilities for signification made available by the Italian language, and with the necessity of paying close attention to how the French text says what it says. I am to Brossard what Maude Laures is to Angstelle. The difference is that Brossard is in both Angstelle and Laures. I start where Brossard/Laures leaves off, and I work my way back to Brossard/Angstelle having already been acquainted with Brossard/ Laures. My approach to Angstelle's text then, is already informed by Laures's approach, specifically, it is informed by the way I make the subjective thrust of my language seep through her angle of vision. Call it identification with a difference. Not all of it is conscious, not all of it is immediately visible.

$>$ At the end of this first part of my project, there are a few interventions of my own desire that I have consciously applied : $>\bullet$ Generally, I have transformed Mélanie in a more self-assertive character. In chapters 4, 5 and 6, Mélanie speaks mostly in the present conditional. In Italian I have transposed most of these verbs in the future indicative, to underscore the urgency and certainty of Mélanie's desire.

$>\cdot$ I have insisted on foregrounding the cinematic quality of most of Brossard's sentences. The (moving) image, as the hologram in Picture Theory, plays a crucial mediating role in the passage from physical perception to linguistic meaning throughout the entire text. Moreover, this passage is one of the primary aspects of translation staged/theorized by the text.

$>$ • Where possible, I have used Italian words whose sound is close to the French original. In her translation, Maude Laures frequently plays on the assonance of signifiers to underscore the dissonance of their semantic connotations. I have attempted to give to the Italian Maude Laures a similar space of phonetic/semantic manoeuvre. However, just what kind of relation will emerge in Italian between Laures and Angstelle will be clear only at the end of this work. 
$>$ The annotations are thus just a provisional guide to the interpretive processes that are presently informing my work. The pattern, I believe, will become clear only at the end.

$>$ Let me add a few more technical details :

$>$ Changes in punctuation patterns tend to be silent. I only signal them in my notes when the changes perceptibly affect semantics.

$>\bullet$ There are some brackets in the text itself containing, in bold, the original French signifiers. They are there to remind me that I need to do more thinking on my translation choices.

$>\bullet$ In some places I felt that my lack of knowledge of Quebecois idiomatic expressions barred me from understanding the passage. I signal those moments with a note that says "check with Brossard."

$>\cdot$ With regards to the presence of English words in the text, I have translated into Italian small syntactical particles - such as "yet," for example - and left in English more substantial strings of words, such as Mélanie's last sentences at the end of the novel. I've done so because frequently the insertion of the English signifier in the French text is contingent on the specific historical relation between Quebecois and English, a relation that is simply not the same between English and Italian, and thus cannot be always and fully reproduced.

>•I frequently make reference to Susanne de Lotbinière-Harwood's English translation of Le désert mauve. Her translation enabled me to understand better some passages. Sometimes I even prefer following in the wake of her own translation. I give a reason for this in my notes.

\section{2) Exemple de traduction avec commentaire et en présence de différentes options}

Il cervello è fragile, sono necessarie molte superstizioni per adunarlo a sé e non inabissare nulla intorno ("Le cerveau est fragile, il faut beaucoup de superstitions pour se le rallier et ne rien abîmer autour". Maude Laures translates: "Il n'est pas facile de substituer les images, d'allier l'abîme et la plénitude en soi", thus playing upon the assonance between "rallier" and "allier" and "abîmer" and "abîme". My translation of "se le rallier" as "adunarlo a sé" and of "abîmer" as "inabissare" attempt to render that play of assonance possible in Italian. My translation of Maude Laures' text will in fact be: "Non è facile sostituire le immagini, alleare in sé l'abisso e la pienezza". There is however a semantic problem when I translate "abîmer" with "inabissare": whereas as "abîmer", although it retains as archaic the meaning of will partially lend itself to that play in Maude Laures" text. I can translate there "all my translation "inabissare" attempts to keep with Brossard's signifier rather than with its meaning, (guastare) (206) late "abîmer" with "inabissare". A mostly means "to ruin, waste, damage contains as archaic the meaning "to sink, cast down in the abyss"; "inabissare" on the other hand, would only retain the archaic meaning of "abîmer" and lose entirely the active connotation of "ruin, damage" which seems to me here to be the main connotation. Maude Laures in her translation in fact plays upon the phonetic assonance of the signifiers in order to foreground a deeper semantic dissonance between them : her translation in fact is focused on making Mélanie's statements more assertive and self-confident, shifting the emphasis away from Angstelle's dramatization of a teenage's moment of rupture with the epistemic and affective field of patriarchy. For Maude Laures Mélanie is, in a sense, already "on the other side", she is well acquainted with the territory of "beauty" or well acquainted enough with the territory of "beauty" to remain relatively untouched by phallogocentric constraints on knowledge and affectivity. In Maude Laures' version Mélanie's crush on Angela Parkins is less about a desire to be like her (a motif very prominent in Angstelle's text) rather than about a recognition that in Angela Parkins there is something she herself (Mélanie) already has : a specific mode of knowledge founded upon the exactitude of passion. Now, to return to our translation problem, the Italian signifier "inabissare" does not stand in semantic dissonance to "abisso" as "abîmer" does to "abîme". If I translate "abîmer" with "guastare" however, I will lose the phonetic assonance which is part of the play of Laures' translation. This kind of issue I believe can only be settled at the end of my work, when I will be able to assess exactly the dominant tone of my translation of Angstelle's text versus the dominant tone of Laures.) Non c’é pi deserto. Grazie non avrà mai quindici anni.

La version définitive renonce au glissement sémantique, mais perd en même temps le jeu d'assonances :

«Il cervello è fragile. C'è bisogno di un bel po' di superstizioni per adunarlo a sé senza danneggiare nulla intorno »(p. 26/38).

« Non è facile sostituire le immagini, alleare l'abisso e la pienezza in sé » (p. 20/18). 


\section{Mercier e Camier, Chiara Montini. Genèse d'une double traduction du français (1) et de l'anglais (2)}

\section{1) Dal francese}

Il viaggio di Mercier et Camier, lo posso raccontare se voglio, perché fui sempre con loro.

Fu un viaggio fisicamente $1 /$ materialmente/concretamente abbastanza facile [di nessuna difficoltà materiale ${ }^{2}$, senza mari né frontiere da superare $^{3}$ - attraversare (franchir), attraverso regioni poco accidentate, anche se desertiche a tratti [anche se deserte per collocazione geografica $^{4}$. Restarono a casa loro, ebbero questa fortuna [occasione ${ }^{5}$ ] inestimabile. Non ebbero da affrontare [cimentarsi], con maggiore o minor successo [fortuna ${ }^{6}$ (bonheur), [con] dei costumi stranieri, una lingua, un codice, un clima e una cucina bizzarri, in un quadro [in ambienti] (décor ${ }^{7}$ ) che poco avesse a che vedere, (n'ayant que peu de rapport), dal punto di vista della somiglianza, con quello cui dapprima la tenera età, poi l'età matura ${ }^{8}$ poi li avevano temprati [in ambienti che avessero, dal punto di vista della somiglianza, un sia pur minimo rapporto ${ }^{9}$ con quello a cui l' infanzia ${ }^{10}$ prima e l' età matura poi li avevano abituati]. Il tempo, benché spesso inclemente (ma c'erano abituati) [Il clima, anche se a volte ${ }^{11}$ inclemente (ma c' erano avvezzi), non uscì [supero'] mai dai limiti del temperato, ovvero di quello che può sopportare senza pericolo se non senza disagio (désagrément), un uomo di quella zona che indossi scarpe e vestiti adatti. Quanto al denaro, se non ne avevano abbastanza per viaggiare in prima classe e per alloggiare (déscendre) negli hotel di lusso (palace12), ne avevano a sufficienza per andare e venire senza tendere la mano. Si può perciò affermare che da questo punto di vista godevano di condizioni favorevoli (meglio di "le condizioni gli erano favorevoli), moderatamente favorevoli [sia pur moderatamente, favorevoli13]. Dovettero lottare, ma meno di molti altri, meno forse della maggior parte delle persone che se ne vanno (s'en vont), [14] spinti da un bisogno talora (talvolta) chiaro, talora oscuro [a volte chiaro, a volte scuro ${ }^{15}$ ].

1. Anche con l'aiuto dell'inglese. Matériellement, Robert, 4 définitions : par rapport à la matière (phil.) 2 D'une manière matérielle, dans le domaine de la matière 3 par rapport au corps, à ce qui concerne le corps. fisicamente 2 materialmente, realmente, concretamente: nella realtà fisica per mezzo dell'esperienza diretta e attraverso i sensi. Materialmente: 2 In base all'esperienza diretta, per mezzo dell'attività pratica, dell'applicazione delle facoltà fisiche, $\mathrm{o}$, anche, delle facoltà sensoriali; sensibilmente, tangibilmente. 3 In base alla realtà oggettiva ed evidente; concretamente, di fatto; certamente; effettivamente, realmente.

2. In corsivo e tra parentesi quadra traduzione di Luigi Buffarini. Qui la traduzione di Bu toglie la vaghezza del testo, precisando cio' che Be non sembra tenere a giustificare.

3. In corsivo quando adotto trad. di Buffarini.

4. Chiarificazione che si allontana dal testo.

5. Occasione è più contingente di fortuna, presuppone la capacità di saperla cogliere anche quando è data dal fato (l'occasione fa l'uomo ladro). Qui Be è più aleatorio, M. et C. non hanno un ruolo attivo.

6. Vedi inglese, success. Bonheur puo' essere usato come " success », nella 2 accezione del Robert: Réussite, succès (avoir plus de bonheur que de mérite) ; Avoir du bonheur dans ses entreprises.

7. Décor (Robert) Aspect extérieur du milieu dans lequel se produit un phénomène, vit un être, considéré sous son aspect extérieur et accidentel. Ambiance, apparence, atmosphère, cadre, décoration, milieu, toile (de fond).

8. Ripetizione di âge.

9. Errore di interpretazione? faux sens Qui vuol dire un quadro che avesse una relazione scarsa in senso positivo e non negativo 'un sia pur minimo rapport' è concessiva, mentre qui si tratta di una consecutiva???

10. Meglio tenere tenera età per conservare l'ironia e la ripetizione ancora più forte in inglese.

11. Souvent, traduce con "a volte", perché?

12. Palace: Hôtel de grand luxe de renom international (Robert) Vedi poi anche l'inglese: palatial hotel (Sansoni, "un albergo di lusso").

13. Si tratta di uno stratagemma letterario cui Beckett ricorre sovente. Ad un'affermazione segue un'attenuazione dell'affermazione stessa - che perde il suo carattere assoluto - e a volte anche una negazione. La virgola che separa l'affermazione dall'avverbio finale è significativa. EPANORThOSe (gr. redressement) figure par laquelle on feint de rétracter ce qu'on vient de dire pour l'exprimer ensuite d'une manière plus vigoureuse (voir correctif, correction) Perciò la traduzione di Buffalini, non rispetta l'intentio operis, ma tende a imbellire. Vedi anche testo inglese: "up to a point". Credo che l'italiano possa, al limite, tollerare la ripetizione di favorevoli.

14. Qui Buffarini sopprime la virgola, che invece sembra segnare una pausa "quelli che se ne vanno" ( come l'autore), spinti da un bisogno talora chiaro talora oscuro.

15. La traduzione di obscur con "scuro", sombre, non corrisponde al testo. Si tratta di oscurità del bisogno, e non di una colorazione. Anche in inglese obscure, riprende il termine francese, e non usa "dark". Qui ho voluto usare "talora", più ricercato di "a volte", per rendere la sonorità di tantôt (forse anche talvolta). 
Si erano consultati lungamente prima di intraprendere questo viaggio, pesando con tutta la calma di cui erano capaci i vantaggi e gli svantaggi che potevano risultarne, per loro. Il lato oscuro, il roseo, a turno li passavano in rassegna. (Si erano consultati a lungo prima di intraprendere questo viaggio, pesando con tutta la calma di cui erano capaci i vantaggi e gli svantaggi che potevano risultarne, e passando in rassegna il lato oscuro e quello roseo ${ }^{16}$.) La sola certezza che ottenevano da questi dibattiti era di non lanciarsi all'avventura con leggerezza. [Si erano consultati a lungo prima di intraprendere questo viaggio, calcolando, con tutta la calma di cui erano capaci, $i$ vantaggi e gli svantaggi che ne potevano risultare. A turno ottimisti e pessimisti, l'unica cosa su cui si trovavano d'accordo 17 era quella di non gettarsi alla leggera nell' avventura]

\section{2) Dall' inglese}

Il viaggio di Mercier e Camier posso raccontarlo, se voglio, poiché fui sempre con loro.

Fisicamente fu abbastanza facile, senza mari né frontiere da attraverare, per regioni prive di intemperie (non tempestose) ${ }^{18}$ nel complesso, per quanto desertiche a tratti (in parts ${ }^{19}$ ). Mercier et Camier non si mossero da casa, ebbero questa inestimabile (great good) fortuna. Non dovettero affrontare, con maggiore o minor successo, modi, lingue, leggi, cieli, cibi esotici (outlandish ${ }^{20}$ ) in ambienti (surroundings ${ }^{21}$ ) poco dissimili da quelli cui dapprima l'infanzia, poi l'adolescenza, poi l'età adulta li avevano temprati ${ }^{22}$. Il tempo, anche se spesso inclemente (ma non conoscevano di meglio), non superò mai i limiti del temperato, ovvero di ciò che una persona normale (average) originaria di quella zona poteva sopportare, senza pericolo se non senza disagio, con i vestiti e le scarpe appropriate. Per quanto riguarda il denaro, se anche non copriva le spese di viaggio in prima classe o in hotel di lusso23, ce n'era comunque a sufficienza da permettergli di andare e venire senza far ricorso all'elemosina (alms). Si può perciò dire che anche in questo caso erano fortunati, fino a un certo punto. Ebbero da lottare, ma meno di molti altri, meno forse della maggior parte di quelli che partono all'avventura (venture forth,) spinti da una necessità ora chiara ora oscura.

Si erano lungamente concertati, prima di intraprendre (embarking24) questo viaggio, pesando con tutta la calma a loro disposizione (at their command ${ }^{25}$ ) quali benefici (benefits) potessero sperare da esso, quali danni (ills) temere, soffermandosi ora (alternando) sui lati oscuri ora su quelli rosei della situazionemantaining turn about the dark side and the rosy. La sola certezza che trassero da queste discussioni fu quella di non gettarsi con leggerezza, nell'ignoto(/nel buio)lunching out, into the unknown26).

16. Questa seconda versione trae ispirazione dal francese e dall'inglese. combinazione delle due. Ma cerco migliore soluzione:

17. "La seule certitude": qui si tratta davvero di certezza, perché, difatti tutto il viaggio è incerto, addirittura non esiste. Et en effet après il s'agit de manque de certitude quand au rendez-vous [rafforzata in inglese dall'affermazione del narratore, assente in francese, "so they had an appointment".

18. Untormented: torment: 1 orig. the infliction or suffering of torture. Later, (the condition of enduring) extreme pain or suffering; physical or mental agony, 2 a cause of extreme pain, suffering or anguish. Now also a source of worry or annoyance; a troublesome person ME 2 a violent storm; a tempest.

19. III region; side. ME in $p l$. A portion of a country or of the world; a region; an area.

20. Outlandish: foreign, alien; not native or indigenous 2 looking or sounding foreign; unfamiliar, strange, odd, unikely, bizarre 3 out-of-the way, remote; far removed from civilization.

21. Surrounding: overflowing, inondation. Pl. surroundings: those things wihich surround or are in the vicinity of a person or thing; the conditions affecting a person or thing; environment.

22. Ripetizione di "hood" tre volte.

23. Alatial: of the nature of a palace; magnificent,splendid; pertaining to or befitting a palace.

24. Sansoni.

25. Sansoni: a loro disposizione.

26. Take a leap into the unknown fare un salto nel buio (e-dizionario, Word Reference). 
Chiara Montini est chercheure associée à l'ITEM (CNRS-ENS) et traductrice. Elle étudie la génétique textuelle, le multilinguisme et la traduction. Elle a enseigné la littérature française et comparée à l'université Paris VIII et à l'université de Provence. Auteure de nombreux articles sur la traduction et le multilinguisme et d'une monographie sur Samuel Beckett, elle a traduit, en italien, plusieurs textes, dont le roman Mercier et Camier de Samuel Beckett.

montini.chiara@gmail.com

\section{Résumés Écrire et décrire la genèse de la traduction. Le Désert mauve et Mercier et Camier}

Le propos de cet article est d'interroger la genèse des traductions en prêtant une attention toute particulière à la dynamique qui s'instaure entre les textes et leurs traductions. Cette dynamique, nous l'entendons au sens que lui donnait Antoine Berman, comme ce qui met en jeu la relation entre le même et l'autre, entre l'écriture (comme traduction), le sujet, et bien sûr les langues. Deux textes servent ici de point d'appui à l'analyse - Le Désert mauve de Nicole Brossard et Mercier et/and Camier de Samuel Beckett - pour montrer que genèse et traduction représentent les multiples facettes, les multiples origines de l'écriture à travers ses métamorphoses et ses possibilités. Comment le traducteur représentera-t-il l'ambiguïté du texte qui s'inscrit dans sa genèse et dans sa traduction au point de constituer un tout unique ? L'article s'efforce de répondre à cette question à partir de la genèse de deux traductions, Il deserto malva, de Elena Basile et Mercier e Camier de Chiara Montini.

This article questions the genesis of translations by focusing in particular on the dynamic that takes place between texts and their translations. We give to the term "dynamic" Antoine Berman's definition, as that which questions the relation between the same and the other, between writing (as translation), the subject and, of course, languages. We will use two texts to support our analysis: Nicole Brossard's Le Désert mauve and Samuel Beckett's Mercier etland Camier, to show that genesis and translation represent the numerous facets and origins of writing, through its metamorphoses and possibilities. How will the translator represent the text's ambiguity which inscribed in its genesis and its translation to the extent that it constitutes a single whole? This article will try to answer this question on the basis of the genesis of two translations: Elena Basile's Il deserto malva and Chiara Montini's Mercier e Camier.

Das Ziel dieses Artikels ist es, die Genese der Übersetzungen zu analysieren und dabei ein besonderes Augenmerk auf die zwischen den Texten und ihren Übersetzungen entstehende Dynamik zu richten. Im Sinne von Antoine Berman wird diese Dynamik als das verstanden, was die Beziehung zwischen dem Selbst und dem Anderen, zwischen dem Schreiben (als Übersetzung), dem Subjekt und, natürlich, den Sprachen infrage stellt. Gestützt auf zwei Texte - Le Désert mauve von Nicole Brossard und Mercier et/and Camier von Samuel Beckett - soll hier aufgezeigt werden, dass Textgenese und Übersetzung die multiplen Facetten und Ursprünge des Schreibens durch seine Metamorphosen und Möglichkeiten wiedergeben. Wie wird der Übersetzer vorgehen, um die Ambiguität zwischen der Genese des Textes und seiner Übersetzung darzustellen und daraus eine stimmige Einheit zu schaffen? Anhand der Genese von zwei italienischen
Übersetzungen der beiden Texte, Il deserto malva von Elena Basile und Mercier e Camier von Chiara Montini, versucht der vorliegende Artikel, Antworten auf diese Frage zu finden.

Este artículo se propone analizar la génesis de las traducciones, poniendo el acento en la dinámica que se instaura entre los textos y sus traducciones, en el sentido que le otorgaba Antoine Berman: dinámica que pone en juego la relación entre lo mismo y lo otro, entre la escritura (como traducción), el sujeto y, por supuesto, las lenguas. El análisis se apoya en dos textos-Le désert mauve de Nicole Brossard y Mercier et/and Camier de Samuel Beckett-, para mostrar que génesis y traducción representan las múltiples facetas, los múltiples orígenes de la escritura a través de sus metamorfosis y de sus posibilidades. ¿Cómo procederá el traductor para representar la ambigüedad del texto que se inscribe en su génesis y en su traducción hasta constituir un todo único? Trataremos de responder a este interrogante basándonos en la génesis de dos traducciones: Il deserto malva, de Elena Basile y Mercier e Camier de Chiara Montini.

Este artigo sobre a génese de traduções presta particular atenção à dinâmica da relação entre o texto e a sua tradução. Uma dinâmica que entendemos no sentido dado por Antoine Berman, de uma relação entre o mesmo e o outro, entre a escrita (como tradução), o conteúdo e, bem entendido, as línguas. Dois textos servem a esta análise - Le Désert mauve de Nicole Brossard e Mercier et/and Camier de Samuel Beckett - para mostrar que génese e tradução representam as múltiplas facetas, as múltiplas origens da escrita através de metamorfoses e potencialidades. Como conseguirá o tradutor representar a ambiguidade do texto que decorre tanto da génese como da tradução, por forma a constituir um todo único? Para responder a esta pergunta, o artigo usa as traduções Il deserto malva, de Elena Basile, e Mercier e Camier de Chiara Montini.

I 1 seguente saggio si propone di analizzare la genesi delle traduzioni insistendo sulla relazione dinamica che si instaura tra i testi e le loro traduzioni. Relazione dinamica nel senso di cui si serve Antoine Berman, che mette in gioco il rapporto tra lo stesso e l'altro, tra la scrittura (come traduzione), il soggetto e ovviamente le lingue. Si è scelto, a tale scopo, di studiare due testi che trattano sia di traduzione sia di genesi: Le Désert mauve di Nicole Brossard e Mercier et/and Camier di Samuel Beckett. In entrambi i testi, genesi e traduzione rappresentano i molteplici aspetti e origini della scrittura attraverso le sue metamorfosi e le sue possibilità. Come procederà il traduttore per rappresentare l'ambiguità del testo che s'iscrive nella sua genesi e nella traduzione a tal punto da costituire un'entità inscindibile? Tenteremo di rispondere a questa domanda ripercorrendo la genesi delle traduzioni italiane dei due testi: Il deserto malva, di Elena Basile e Mercier e Camier di Chiara Montini. 